\title{
Le contrôle de l'immigration en Grèce dans les années 90
}

Anastassia Tsoukala

\section{CpenEdition}

\section{Journals}

Édition électronique

URL : http://journals.openedition.org/conflits/363

DOI : $10.4000 /$ conflits.363

ISSN : $1777-5345$

Éditeur :

CCLS - Centre d'études sur les conflits lilberté et sécurité, L'Harmattan

Édition imprimée

Date de publication : 15 octobre 1997

ISSN : 1157-996X

Référence électronique

Anastassia Tsoukala, «Le contrôle de l'immigration en Grèce dans les années 90 », Cultures \& Conflits [En ligne], 26-27 | automne 1997, mis en ligne le 15 mars 2006, consulté le 30 mars 2021. URL : http:// journals.openedition.org/conflits/363 ; DOI : https://doi.org/10.4000/conflits.363

Ce document a été généré automatiquement le 30 mars 2021.

Creative Commons License 


\title{
Le contrôle de l'immigration en Grèce dans les années 90
}

\author{
Anastassia Tsoukala
}

\section{Anastassia TSOUKALA ${ }^{1}$}

Introduction Longtemps considérée comme un pays d'émigration, la Grèce a assisté, dès les années soixante-dix, à un flux d'immigration croissant qui, ayant acquis des dimensions considérables dès le début des années quatre-vingt, la plaçait, en 1993, au troisième rang, en Europe occidentale, quant au taux d'immigrés résidant de manière régulière ou non sur son territoire ${ }^{2}$ et, en 1996, au premier rang, dans l'Union européenne, quant au taux d'immigrants clandestins ${ }^{3}$. L'impact de cette hausse des immigrés étrangers ne saurait pourtant être entièrement saisi si nous ne tenons pas compte de l'arrivée, par vagues successives, tout au long de l'après-guerre, de plusieurs centaines de milliers d'immigrants d'origine grecque qui, selon leur statut socioéconomique, rendaient plus ou moins manifeste l'absence de toute politique d'intégration. À cette absence s'ajoutaient l'inefficacité des stratégies d'action policières appliquées dans la lutte contre l'immigration clandestine ${ }^{4}$ et les faiblesses d'un cadre normatif dépassé car, jusqu'au début des années quatre-vingt-dix, la politique grecque sur l'immigration se fondait essentiellement sur une loi adoptée en 1929. Cherchant alors à remédier à ces faiblesses et à se conformer aux lignes directrices de la politique adoptée en la matière au niveau européen, le législateur a adopté, en 1991, la loi $1975^{5}$ où, tout en prévoyant les premières mesures sociales pour les immigrés et en assurant une meilleure protection de leurs droits, il se consacre à la lutte contre l'immigration clandestine. D'après le ministère de l'Ordre Public, celle-ci est censée contribuer, d'une part, à l'exploitation des étrangers et, par conséquent, à l'essor de l'économie parallèle et, d'autre part, à la hausse de la délinquance et du chômage, à l'émergence de réactions racistes et xénophobes de la part de la population ${ }^{6}$ ainsi qu'à la provocation de tensions éventuelles dans le Nord du pays, où vit la minorité musulmane grecque. La mise en évidence des options de cette nouvelle politique nécessite la présentation de ses éléments constitutifs. Par conséquent, nous examinerons, dans un premier temps, l'ampleur du phénomène et les réponses qu'il a 
suscitées afin de pouvoir saisir les tendances et les logiques sous-tendant la conception et la mise en application de cette politique. La formation de la politique L'ampleur du phénomène Malgré le problème de fiabilité des statistiques, dû, dans le passé, à des faiblesses administratives et, à présent, à la forte hausse de l'immigration clandestine, nous pouvons apercevoir les principales tendances de l'immigration en Grèce, relatives aux immigrants d'origine grecque ou étrangère. Les immigrants d'origine grecque Parmi les flux migratoires qui ont jalonné toute l'histoire grecque de l'après-guerre, nous citons :

- l'arrivée, dans les années cinquante, d'environ 200000 membres des minorités grecques en provenance de Turquie et d'Égypte ;

- le retour des réfugiés politiques grecs. Il s'agit d'environ 56000 personnes qui, ayant dû quitter le pays après la fin de la guerre civile de 1946-49, se sont installées dans l'exUnion soviétique et dans les pays de l'Europe de l'Est. Rentrés en Grèce surtout après 1974, en 1984 ils atteignaient 33500 personnes $^{7}$;

- l'arrivée des Grecs de la diaspora dans l'ex-Union soviétique, dits Pontiques ${ }^{8}$. Ils ont commencé à immigrer à partir de 1987, surtout après 1989, et en 1996 ils atteignaient près de 70000 personnes $^{9}$, les autorités prévoyant, pour les années suivantes, l'arrivée annuelle de 12-15 000 personnes ${ }^{10}$. Leur présence en Grèce a suscité ce que nous pourrions considérer comme la première mise en œuvre d'une politique d'intégration ; - l'arrivée des membres de la minorité grecque en Albanie, estimée à 400000 personnes, selon les représentants de cette communauté et des sources officieuses du gouvernement grec, ou à 200000 personnes, selon le World Directory of Minorities, ou à 58000 personnes, selon les statistiques albanaises ${ }^{11}$. Vu que, dès le début des années quatre-vingt-dix, le gouvernement grec avait pris à leur égard une position particulière, justifiée par des motifs économiques aussi bien que politiques, qui consistait à les considérer comme des demandeurs d'asile, ils étaient le plus souvent des immigrants clandestins. Estimés à 70000 en 1991, ils auraient atteint 150000 personnes en 1996, selon le ministère du Travail grec. En 1995, le gouvernement grec a commencé à leur délivrer des visas de cinq ans qui sont aussi valables comme cartes de séjour pourvu que leurs titulaires retournent en Albanie au moins une fois par semestre. Il faut pourtant souligner que, jusqu'à présent, l'étendue de l'application de cette mesure est restée assez limitée, le gouvernement continuant en fait à être réticent à l'installation massive de cette minorité en Grèce. Les immigrants étrangers Les étrangers en situation régulière : En hausse constante dès les années soixante-dix, en 1994 ils atteignaient près de 244000 personnes, dont environ 66000 étaient des ressortissants des pays membres de l'Union européenne - les autres étant notamment originaires des pays du tiers-monde et de l'Europe de l'Est ${ }^{12}$. Les étrangers en situation irrégulière : Bien manifeste dans les années quatre-vingt, l'immigration clandestine a récemment connu une forte hausse, due surtout à l'arrivée massive, dès 1991, d'immigrants albanais. Plus précisément, les immigrants clandestins, estimés à 19000 en $1980^{13}$ et à 70-105 000 en $1988^{14}$, s'élèveraient à 363000 en 1993 , dont 150000 Albanais, 100000 Polonais, 55000 Égyptiens, 40000 Philippins, 13000 Iraniens et 5000 de nationalités autres ${ }^{15}$. Bien qu'impressionnantes, ces estimations restent inférieures à celles du ministère de l'Ordre Public, selon lequel il y aurait en 1993 près de 500000 immigrants clandestins en Grèce, dont environ 200000 Albanais ${ }^{16}$. D'après d'autres estimations, faites tant du côté grec que du côté albanais, le nombre des immigrants albanais présents en Grèce en 1993 serait de $300000^{17}$. La crise suscitée par la faillite de plusieurs sociétés financières en Albanie n'ayant pas provoqué un exode massif des 
Albanais vers la Grèce, il y aurait à présent, selon le ministère du Travail, 468000 immigrants clandestins, correspondant à environ $4,5 \%$ de la population du pays, dont : 200000 Albanais, 88000 Polonais, 32000 Russes, 20000 Iraqiens, 14000 Égyptiens, 10 000 Bulgares, 10000 Roumains, 8000 Philippins, 6000 Turcs, 6000 ressortissants de l'ex-Yougoslavie, 5000 Iraniens, 5000 Syriens, 5000 Pakistanais, 5000 Indiens, 4000 Éthiopiens et 50000 de nationalités autres. Par ailleurs, nous devons souligner que, par sa position géographique et par la morphologie de ses frontières, la Grèce constitue un terrain propice à la prolifération des réseaux de trafic d'immigrés, dirigés tant par des étrangers que par des $\mathrm{Grecs}^{18}$. Basés surtout en Turquie, au Liban et dans les pays de l'Afrique du Nord, ces réseaux qui, jusqu'aux années quatre-vingt, étaient d'une portée limitée (5-20 immigrants transportés par trajet) ont commencé à se développer vers la fin des années quatre-vingt et, actuellement, leurs dirigeants organisent des transports de plus de 150 immigrants par trajet - choisissant de préférence comme point d'entrée une des îles grecques ${ }^{19} .{ }^{20}$ Les demandeurs d'asile : Ayant signé la Convention de Genève sous certaines réserves, jusqu'au milieu des années quatre-vingt-dix la Grèce n'offrait pas aux réfugiés le droit de travailler ou de résider de manière permanente sur son territoire. Suite pourtant à l'adoption du décret présidentiel 209/1994 ${ }^{21}$, la Grèce a cessé d'être un pays de transit et les réfugiés sont à présent autorisés à y résider et $\mathrm{y}$ travailler de manière permanente. Si, jusqu'au début des années quatre-vingt, le nombre de demandeurs d'asile était peu élevé, entre 1983 et 1990 nous assistons à une hausse annuelle de 52,7\% ${ }^{22}$. Entre 1980 et 1993 la Grèce a reçu 14604 demandes d'asile déposées, dans $80 \%$ des cas, par des ressortissants turcs, iraqiens, polonais, iraniens, roumains et albanais, mais n'a reconnu la qualité de réfugié qu'à 2889 personnes $^{23}$. En 1994, le nombre de demandes d'asile s'élevait à 1302 et, en 1995, à 1312 mais la qualité de réfugié n'a été reconnue qu'à 170 et à 203 personnes respectivement ${ }^{24}$. Le nombre de réfugiés s'élève à présent à environ 5000 personnes (y sont inclus les réfugiés qui sont sous le mandat du HCR) qui, dans la plupart des cas, sont des ressortissants iraqiens, turcs et iraniens. Nous devons enfin signaler que, jusqu'à 1996, les demandeurs d'asile ne faisaient l'objet d'aucune mesure privative de liberté. Ils devaient seulement rester à la disposition des autorités, jusqu'à ce que leur demande soit examinée, la loi ne leur interdisant que le changement d'adresse déclarée ou désignée par les autorités avant la notification de la décision prise par le comité compétent en la matière. Cette attitude tolérante a été profondément modifiée par l'adoption, en décembre 1996, de la loi $2452^{25}$. Celle-ci prévoit la création de " zones d'attente ", aux ports et aux aéroports utilisés comme points d'entrée au pays, où les demandeurs d'asile doivent rester jusqu'à ce que leur demande soit examinée. La durée maximale de cette attente est fixée à quinze jours. Mais, bien que cette loi accorde aux représentants du HCR le droit d'accès à ces zones, elle reste, à nos yeux, assez contestable car elle ne reconnaît aux demandeurs d'asile ni le droit à un recours devant les instances judiciaires ni le droit à un interprète. Nous devons toutefois souligner que, en réalité, la création de ces " zones d'attente " ne concernera qu'un nombre limité de demandeurs d'asile car, dans la plupart des cas, ceux-ci n'arrivent pas en Grèce par avion ou par transport maritime. Les réponses au phénomène Toute la politique grecque sur l'immigration ayant été sous-tendue par le principe que la Grèce n'est pas un pays d'immigration, l'adoption et la mise en application de la loi 1975/1991, modifiée par la loi 2452/1996, ont marqué, à plusieurs égards, un durcissement de cette politique ${ }^{26}$. Ceci nous paraît visible tant en matière d'entrée et d'éloignement des étrangers non communautaires qu'en matière de sanctions prévues en vue de combattre l'immigration clandestine. Les réponses 
législatives L'entrée: Reprenant essentiellement les dispositions de la législation antérieure, le législateur prévoit que tout étranger souhaitant entrer en Grèce doit présenter les documents et visas requis par les conventions internationales et par la législation en vigueur, les garanties de son rapatriement et les justificatifs de ses moyens d'existence ainsi que, le cas échéant, de ceux de sa famille. Par ailleurs, le législateur refuse l'entrée à tout étranger atteint d'une maladie considérée comme susceptible de mettre en jeu la santé publique ${ }^{27}$, à tout étranger qui, entrant en transit, n'a pas de visa, s'il est requis, et de titre de transport vers un pays autre, ou à tout étranger qui, entrant en Grèce en vue d'y travailler, n'est pas muni d'une autorisation préalable $^{28}$. Soucieux, en outre, d'assurer davantage le maintien de l'ordre et de la sécurité, le législateur innove en introduisant la liste de persona non grata. Plus précisément, il élargit le champ d'application des dispositions de la législation antérieure qui, dans ce cas, n'interdisaient l'entrée qu'aux étrangers déjà expulsés du pays, et prévoit l'établissement de cette liste, laquelle, relevant de la compétence du ministère de l'Ordre Public, comprend : i) les étrangers qui, suite à une violation de la législation en vigueur, ont fait l'objet de n'importe quelle mesure d'éloignement ; ii) les étrangers qui, en vertu de la législation en vigueur, sont passibles d'une expulsion administrative, notamment s'ils sont considérés comme menaçants pour l'ordre public, pour la sûreté nationale ou pour la santé publique. À cette liste s'ajoutent enfin les listes de persona non grata dressées au niveau communautaire ainsi que celles dressées par des pays ayant conclu des accords bilatéraux avec la Grèce. Le législateur refuse aussi l'entrée à tout étranger qui, d'après les circonstances, peut raisonnablement être considéré comme immigrant clandestin ou comme dangereux pour l'ordre public ou pour la sûreté nationale. En outre, il prévoit que tout étranger qui se voit opposer un refus d'entrée doit quitter le pays dans le plus court délai possible sinon il fait l'objet d'une reconduite à la frontière - l'étranger ne disposant pas de voie de recours. En cas de demandeur d'asile, si la demande est jugée manifestement infondée ou si elle est déposée par une personne originaire d'un pays tiers " sûr ", elle est examinée de manière prioritaire et sommaire. La même procédure est appliquée si cette demande est déposée lors de l'arrivée de l'étranger à un port ou aéroport utilisé comme point d'entrée au pays, les autorités devant se prononcer dans les quinze jours de l'entrée du demandeur en Grèce, qui, pendant ce délai, reste dans la " zone d'attente ". Enfin, en cas de déposition lors de l'arrivée de l'étranger à un autre point d'entrée au pays, si la demande est jugée infondée l'intéressé a le droit à un recours devant le chef du service compétent du ministère de l'Ordre Public, qui doit se prononcer dans les cinq jours de la réception du recours - le demandeur d'asile reste, pendant ce délai, à la disposition des autorités. Exceptionnellement, le ministre peut, pour des raisons humanitaires, autoriser le séjour provisoire d'un étranger dont la demande d'asile a été définitivement rejetée, jusqu'à ce qu'il puisse quitter le pays - le législateur introduisant ainsi la notion de réfugié de facto. Enfin, la loi prévoit la possibilité d'accorder une protection provisoire à certaines catégories d'étrangers qui se réfugient en Grèce en cas de force majeure. L'éloignement du territoire: ${ }^{*}$ la reconduite à la frontière La reconduite à la frontière, qui occupait une place mineure dans la législation antérieure, constitue à présent une sanction courante car elle est prévue en tant que peine alternative à la prison en cas d'entrée ou de sortie irrégulière. Cette sanction est aussi prévue en cas de refus de l'étranger, entré en transit ou opposé à un refus d'entrée, de quitter le pays dans le plus court délai possible. Dans tous les cas, l'étranger ne dispose pas de voie de recours. ${ }^{*}$ l'expulsion Le droit grec prévoit deux 
types d'expulsion, l'une judiciaire, prononcée par la cour, et l'autre administrative, ordonnée par le préfet de police. L'expulsion judiciaire : selon les dispositions du code pénal grec, tout étranger condamné à une peine de prison peut être passible d'une expulsion applicable à l'expiration de sa peine d'emprisonnement ou, le cas échéant, aussitôt après sa libération conditionnelle. La cour peut aussi appliquer cette sanction à tout étranger condamné à une mesure de sûreté - l'expulsion est alors prononcée de manière alternative ou cumulative. Ces décisions étant susceptibles d'appel, l'étranger qui a fait l'objet d'une expulsion judiciaire est en outre frappé d'une interdiction du territoire de trois ans. L'expulsion administrative : selon la législation antérieure, tout étranger violant une de ses dispositions pouvait faire l'objet d'une expulsion administrative, ordonnée par le ministre de l'Intérieur qui, si l'étranger cherchait à s'y soustraire, pouvait en outre ordonner sa rétention. Si l'exécution de l'expulsion n'était pas possible, l'étranger pouvait faire l'objet d'une déportation administrative à l'intérieur du pays. Dans tous les cas, l'étranger ne disposait pas de voie de recours. Tout en modifiant radicalement les conditions d'application de l'expulsion, la loi de 1991 a, d'une part, assuré la protection des droits de l'étranger et, d'autre part, imposé certaines contraintes aux instances administratives. Plus précisément, en ce qui concerne les conditions d'application, l'expulsion administrative, toujours applicable à tout étranger qui se voit inscrit sur la liste de persona non grata ou qui ne dépose pas de demande de prolongation de ses permis de séjour et de travail, s'il ne quitte pas le pays dans les délais fixés par la loi, devient facultative :

- si l'étranger est condamné à une peine de prison et ne fait pas l'objet d'une expulsion judiciaire ;

- en cas de violation de la loi 1975/1991;

- si la présence de l'étranger est considérée comme dangereuse pour l'ordre public, pour la sûreté nationale ou pour la santé publique ;

- si l'étranger se présente chaque fois devant les autorités sous une identité différente. L'expulsion, applicable, le cas échéant, à l'expiration de la peine d'emprisonnement de l'étranger ou aussitôt après sa libération conditionnelle, est ordonnée par le préfet. L'étranger peut faire prévaloir ses droits à toutes les étapes de la procédure administrative, sa protection étant renforcée s'il est titulaire d'un permis de séjour de deux ans au minimum ou s'il est membre d'une famille grecque. En ce qui concerne la mise en application de l'expulsion administrative, le ministre de l'Ordre Public peut, en cas d'intérêt public ou si l'étranger est considéré comme dangereux pour l'ordre public ou soupçonné de vouloir se soustraire à l'exécution de son expulsion, ordonner sa rétention. Les personnes concernées sont retenues aux dépôts des commissariats ou de la Préfecture d'Athènes et, bien que ces dépôts soient surpeuplés, la durée de la rétention peut parfois excéder les six mois ${ }^{29}$. Si l'exécution de l'expulsion n'est pas immédiatement possible, le ministre de l'Ordre Public peut autoriser l'étranger à séjourner provisoirement en Grèce tout en lui imposant certaines restrictions portant sur son installation, ses déplacements... N'autorisant pas enfin l'expulsion d'un étranger, auquel a été reconnu le statut de réfugié, vers un pays où il risque d'être persécuté, le législateur prévoit certains cas, liés à l'âge ou aux relations de l'étranger avec une famille grecque, où l'expulsion est toujours interdite. Les sanctions : Soucieux de manifester clairement sa volonté de réprimer l'immigration clandestine, le législateur prévoit pour la première fois des sanctions à l'encontre de tout étranger entrant de manière irrégulière en Grèce, qui est passible d'une peine de prison de trois mois à cinq ans ${ }^{30}$, ainsi qu'à l'encontre de tout étranger travaillant sans permis de 
travail, qui est passible d'une peine de prison de dix jours à cinq ans et d'une amende. En outre, comme la législation antérieure ne sanctionnait que les personnes impliquées au transport d'immigrants clandestins, le législateur renforce à présent ces sanctions et prévoit des mesures répressives à l'encontre des personnes qui emploient des étrangers non munis de permis de travail. Plus précisément, toute personne impliquée au transport d'immigrants clandestins est passible d'une peine de prison d'un an au minimum et d'une amende ; les moyens de transport utilisés à cette fin sont par ailleurs confisqués. En cas de circonstances aggravantes, l'accusé est passible d'une peine de prison de deux ans au minimum et d'une amende cinq fois plus lourde que l'amende initiale. Enfin, toute personne qui emploie un étranger non muni de permis de travail est passible d'une peine de prison de trois mois au minimum et d'une amende. Les réponses policières En vertu de la législation en vigueur, le contrôle des frontières relève de la compétence du ministère de l'Ordre Public mais son exercice est également assuré par les ministères de la Défense et de la Marine Marchande. Plus précisément, les frontières terrestres sont contrôlées par la police et l'armée tandis que les frontières maritimes sont contrôlées par la police, la police portuaire et l'armée. Tout étranger arrêté par des militaires ou par des agents de la police portuaire doit être remis, dans des délais raisonnablement courts, au service compétent du ministère de l'Ordre Public. Afin de renforcer le contrôle des frontières, la loi de 1991 prévoit la constitution d'un corps de police spécial qui, doté d'un équipement plus moderne, augmenterait les effectifs policiers chargés de cette tâche. En août 1994 ont en fait été créées treize unités mobiles, qui ont permis la hausse du nombre de barrages de police et la formation d'une deuxième zone de contrôle des personnes et des véhicules aux régions frontalières. En décembre 1995 a été décidée l'installation à la frontière grécoalbanaise d'un système de surveillance électronique et a été achevée la modernisation de l'équipement des préfectures de police des régions frontalières. L'installation du système de surveillance électronique de la frontière gréco-albanaise n'ayant pas été effectuée jusqu'à nos jours, les options du ministère de l'Ordre Public semblent être de plus en plus orientées vers l'augmentation des effectifs policiers chargés du contrôle des frontières. En effet, en septembre 1996 a été décidé l'envoi à Corfou d'une unité spéciale, composée de 50 policiers chargés de combattre les activités de la mafia albanaise à l'île et de contrôler l'immigration clandestine. En janvier 1997, le ministre de l'Ordre Public a annoncé la préparation d'un projet de loi prévoyant la constitution d'un corps de gardes frontaliers. Composé d'environ 1000 gardes, qui se substitueront aux 700 policiers des unités mobiles précitées, ce corps serait chargé : a) de la prise de toute mesure susceptible d'empêcher l'entrée des immigrés clandestins au pays; $b$ ) de l'arrestation et de la reconduite à la frontière des immigrés clandestins; c) de l'arrestation de toute personne impliquée au trafic d'immigrés clandestins, et d) du concours aux forces armées en cas de conflit ou de mobilisation. Pouvant agir dans tous les départements frontaliers du pays, ce corps ne serait pas opérationnel avant l'automne 1997. Afin de faire face à la hausse des activités des délinquants albanais aux côtes ouest et des trafiquants d'immigrés clandestins turcs aux côtes est du pays, le gouvernement envisage à présent le renforcement des effectifs de la police portuaire et l'achat de nouvelles vedettes, et demande l'implication provisoire des douaniers lors du contrôle des frontières maritimes. Enfin, la Grèce, qui, en vue de la ratification de la Convention d'application de l'accord de Schengen, est tenue de doter d'un équipement électronique 65 au moins de ses 98 points d'entrée, en a équipé jusqu'à présent environ 60 - ceux-ci couvrant près de $90 \%$ des entrées et des sorties du pays. Si, jusqu'aux 
années quatre-vingt, la police était souvent critiquée pour son manque d'efficacité en matière de répression de l'immigration clandestine, dès le début des années quatrevingt-dix ses opérations ont eu des résultats impressionnants. Ainsi, en 1991 ont été reconduits à la frontière 84259 Albanais; en 1992, le nombre d'étrangers reconduits à la frontière s'élevait à 281 643, dont 276656 Albanais et, en 1993, ce nombre était d'environ 229000 étrangers, dont 220655 Albanais. En 1994, la police a reconduit à la frontière 224905 étrangers, dont 216449 Albanais; le nombre d'étrangers reconduits à la frontière en 1995 et 1996 s'élevait à 534 589, dont 512517 Albanais. Le nombre de 12 291 étrangers expulsés entre 1987 et $1990^{31}$ paraît actuellement bien modeste puisque, seulement en 1993, ont été expulsés 9179 étrangers. En 1994 leur nombre s'éleva à 10 601 et, en 1995, à 13 612. Entre 1993 et 1996, la police a arrêté 2055 personnes, d'origine grecque ou étrangère, impliquées au trafic d'immigrés, et a confisqué 1786 moyens de transport utilisés à cette fin. Durant la même période, la police a arrêté 4062 personnes employant des étrangers non munis de permis de travail. En analysant ces statistiques, nous devons d'abord souligner que la hausse des reconduites à la frontière est en grande partie due au fait que beaucoup d'Albanais reconduits à la frontière retournent aussitôt en Grèce - la même personne pouvant ainsi faire l'objet de nombreuses reconduites à la frontière par an. Il faut aussi noter que ces résultats des opérations policières ne correspondent pas toujours à une amélioration du contrôle des frontières, dont les particularités morphologiques les rendent peu contrôlables, car ils reflètent souvent la hausse des contrôles effectués à l'intérieur du pays qui, en décembre 1991, en juin et en octobre 1993 et en août 1994 avaient pris la forme de rafles ${ }^{32}$. Si la prépondérance de ces contrôles nous indique que la police tendait, dans la première moitié des années quatre-vingt-dix, à fonder sa politique sur des contrôles effectués à l'encontre des étrangers déjà présents sur le territoire, la mise en œuvre de ces rafles obéissait plutôt à des critères politiques et économiques, comme nous verrons plus loin. Ce déplacement spatial du contrôle de l'immigration clandestine devient manifeste si nous examinons, par exemple, le nombre d'immigrants albanais arrêtés à la frontière depuis 1991. Nous constatons en effet qu'en 1991 n'y ont été arrêtés que 3052 immigrants, en 19927 515, en 199319302 et, dans les neuf premiers mois de 1994, 6 349. Cette tendance a pourtant été profondément modifiée suite à la formation d'une deuxième zone de contrôle des personnes et des véhicules aux régions frontalières. En effet, entre août 1994 et décembre 1996, ont été arrêtés à la frontière 240689 immigrants, dont 198864 Albanais. La loi conférant enfin un grand pouvoir discrétionnaire à la police, celle-ci tend à appliquer la reconduite à la frontière plutôt que l'expulsion car, d'exécution plus rapide, cette sanction n'est pas susceptible de recours. Appliquée notamment à l'encontre des immigrants albanais, cette pratique policière devient manifeste lorsque nous constatons, par exemple, que parmi les 10601 étrangers expulsés en 1994 il n'y avait aucun Albanais. Le principe selon lequel la Grèce n'est pas un pays d'immigration ayant largement contribué au manque de cohérence et d'efficacité des réponses policières antérieures, les responsables en matière d'immigration renforcent à présent leurs contacts avec leurs homologues européens et cherchent à rationaliser davantage la mise en œuvre de leur politique en fondant de plus en plus leurs stratégies d'action sur la coopération intergouvernementale. La rationalisation: Un Rapport officiel, publié en mars 1992, fixait comme objectifs policiers :

- l'établissement de zones géographiques " dangereuses " car constituant des réservoirs d'immigrants clandestins potentiels ; 
- la localisation des régions d'où partent, habituellement, les immigrants clandestins ;

- l'enregistrement de tous les subterfuges mis en œuvre par les immigrants clandestins ;

- la hausse des effectifs policiers;

- l'amélioration de l'infrastructure des postes de contrôle frontaliers;

- la modernisation de l'équipement utilisé lors du contrôle des frontières (p. ex., usage d'hélicoptères) ${ }^{33}$. La coopération: Selon le même Rapport, le renforcement de la coopération intergouvernementale souhaité devrait permettre :

- le développement du early warning system ;

- l'harmonisation, au niveau communautaire, des politiques à suivre ;

- l'homogénéisation, au niveau communautaire, des modes de rapatriement des immigrants clandestins ;

- l'harmonisation, au niveau communautaire, de la définition de certains termes, tels que " autorités compétentes " ou " entrée irrégulière constatée ";

- l'échange de renseignements ;

- la création d'un service de renseignements central, au niveau communautaire, disposant d'un fichier informatisé ${ }^{34}$. La plupart des objectifs liés à la rationalisation de la mise en œuvre de la politique policière des années quatre-vingt-dix ayant été actuellement atteints, la mise en place de la coopération intergouvernementale nous paraît bien illustrée par l'établissement, dès 1993, de concertations de haut niveau entre la Grèce et la Turquie en vue de combattre le trafic d'immigrés. Il faut en outre souligner que la Grèce a jusqu'à présent conclu ou est en train de conclure des accords de réadmission avec une douzaine de pays, auxquels, à l'exception de la Turquie, sont inclus tous les pays limitrophes. L'application de l'option de coopération s'appuie également en grande partie sur la mise en œuvre des accords de coopération policière conclus avec certains pays, comme fut le cas avec l'Albanie en 1993, mais n'a pas encore impliqué la création d'un réseau d'officiers de liaison en matière d'immigration. Par ailleurs, les rédacteurs du Rapport précité souhaitent que la coopération soit aussi établie entre les différents services nationaux concernés et recommandent le renforcement du contrôle effectué par les services consulaires compétents ${ }^{35}$. En août 1994 a été effectivement établie une coopération entre le ministère des Affaires Étrangères et les consulats grecs en Albanie qui, allant de pair avec la mise en place d'un système de contrôle informatisé, a permis, dans un premier temps, la baisse du nombre de faux visas présentés aux autorités grecques. Comme l'application de cette option de coopération nécessitait un niveau de coordination supérieur, en 1995 a été créé à Corfou un organe de coordination des services publics chargés de la répression de l'immigration clandestine. Ce renforcement du contrôle exercé par les services consulaires grecs n'a pas pourtant empêché la prolifération de réseaux de trafic de visas auxquels, selon des dénonciations faites par les médias grecs, se trouvaient impliqués des membres du personnel administratif des consulats grecs dans plusieurs pays de l'Europe de l'Est, du Moyen Orient et de l'Asie. Cherchant alors à restaurer l'image ternie de ses services consulaires, en février 1997 le ministère des Affaires Étrangères a décidé de rappeler une cinquantaine des fonctionnaires employés dans des consulats et des ambassades grecs en Europe de l'Est. Une interprétation de la politique La présentation du cadre normatif et de la mise en application des réponses étatiques à l'immigration nous a permis de saisir une partie de leurs tendances et logiques sousjacentes. Nous ne saurions pourtant avancer une interprétation de cette politique si nous ne tenons pas compte de l'ampleur et des particularités de l'immigration en Grèce 
ainsi que de la situation politique et économique dans les Balkans. Ceci dit, cette politique nous semble axée sur trois options distinctes : le souci du maintien de l'ordre et de la sécurité, au niveau national, le souci de l'harmonisation, au niveau européen, et le souci de la protection de certains intérêts politiques et socio-économiques grecs, au niveau balkanique. Le souci du maintien de l'ordre et de la sécurité Bien que l'immigration n'occupe qu'une place minime dans le discours politique des années quatre-vingt-dix, la hausse du nombre d'immigrants dans les années quatre-vingt et d'immigrants clandestins dans les années quatre-vingt-dix a rendu impérieuses la modernisation de la législation et l'adoption de stratégies d'action policières appropriées en vue d'assurer le maintien de l'ordre public et de répondre aux pressions de l'opinion publique, qui était de plus en plus inquiète par la hausse de la criminalité dans les années quatre-vingt-dix. Quoiqu'incontestablement amplifiée par les médias ${ }^{36}$, cette hausse de la criminalité, liée le plus souvent aux immigrants albanais, est loin d'être fictive tant dans la région frontalière gréco-albanaise, où se sont notamment développés la contrebande, le trafic de stupéfiants et, récemment, le trafic d'armes, que dans les agglomérations du pays. Plus précisément, les crimes et délits commis par des étrangers en Grèce sont passés de 3 968, en 1990, à 11 750, en 1995, dont une grande partie a été commise par des Albanais. Bien que la participation des étrangers à toutes les infractions constatées pendant cette période reste plutôt faible ou, tout au moins, proportionnée à leur volume, leur taux de participation aux crimes et délits relativement graves est assez élevé ${ }^{37}$, ayant ainsi contribué à une forte hausse du sentiment d'insécurité de la société grecque ${ }^{38}$. Certes, cette montée de l'insécurité et l'hostilité qu'elle impliqua à l'égard des immigrants albanais ne saurait être dissociée de la hausse générale du nationalisme et de la xénophobie constatée en Grèce ces dernières années ${ }^{39}$ mais, en l'occurrence, elle nous paraît aussi attribuable aux tensions existant entre les deux pays. Par ailleurs, nous devons souligner que même si, incontestablement, les méthodes appliquées par la police dans la lutte contre l'immigration clandestine visent avant tout à assurer le maintien de l'ordre public, leur mise en œuvre nous semble obéir en même temps à une autre logique, de nature économique. Nous estimons en effet que, bien que parfois dénoncés pour corruption, les policiers subordonnent moins leurs opérations à la satisfaction de leurs propres besoins matériels qu'à celle des besoins des marchés du travail locaux, comme nous l'indique le fait que les dernières des rafles mentionnées ci-dessus ont eu principalement lieu dans des régions où le marché du travail local n'arrivait pas à absorber les immigrants clandestins. Cette hypothèse nous paraît aussi confirmée par la faiblesse des résultats obtenus en matière de répression de l'emploi des travailleurs étrangers clandestins, le nombre d'arrestations effectuées à cette fin ne correspondant guère à l'ampleur réelle du phénomène, ce qui ne saurait refléter une véritable volonté de combattre l'impact de l'immigration clandestine sur l'essor de l'économie parallèle. Le souci de l'harmonisation À ces pressions internes s'est ajoutée une pression externe, puisque le gouvernement grec avait souvent été critiqué pour les faiblesses de sa politique sur l'immigration. Nous devons souligner à cet égard que, lors de la préparation de la loi 1975/1991, la Grèce n'avait pas obtenu le statut d'observateur de la Convention d'application de l'accord de Schengen. L'ayant obtenu en 1992, elle a adopté depuis une position bien réservée à l'égard de cette convention en reportant constamment sa ratification. Formellement attribuée à l'absence d'un texte de loi sur la protection des données, cette position n'a été abandonnée qu'en 1997, l'adoption de ce texte de loi marquant en fait un tournant décisif de la politique de la Grèce, qui devrait 
ratifier la Convention avant ce mois d'octobre. Soucieux alors d'harmoniser, déjà en 1991, ses options avec celles dominantes en la matière au niveau européen, le législateur a redéfini ses objectifs suivant deux tendances distinctes : l'une sécuritaire, liée à la hausse de la préoccupation manifestée, au niveau européen, à l'égard des questions relatives à l'ordre et à la sécurité, et l'autre libérale, liée à la hausse de l'attention accordée, au niveau international, au respect des droits de l'homme. La tendance sécuritaire Bien manifeste dans le renforcement du pouvoir exécutif, notamment en ce qui concerne l'établissement de la liste de persona non grata et l'élargissement des conditions d'application de l'expulsion administrative, ainsi que dans la place accordée aux instances judiciaires, limitées à une fonction exclusivement répressive, cette tendance détermine en outre le statut de l'étranger qui, privé dans la plupart des cas de ses droits, reste particulièrement vulnérable et fait l'objet de nombreux glissements vers le contrôle de la déviance. Observée ci-dessus en matière d'entrée et d'éloignement des étrangers, cette vulnérabilité atteint son sommet lorsque le législateur interdit aux autorités et aux services publics de prendre en considération toute demande déposée par un immigrant clandestin ${ }^{40}$. Il faut souligner que l'application de cette disposition qui, de toute manière, est contraire à l'article 10 de la Constitution, lequel garantit à toute personne présente sur le territoire grec le droit de s'adresser aux autorités, crée un problème juridique et social majeur, lié notamment à la présence en Grèce de nombreux mineurs albanais impliqués dans des réseaux de prostitution $^{41}$. Intégrée dans la tendance répressive qui caractérisait la législation antérieure, cette tendance sécuritaire s'inscrit en même temps dans celle observée au niveau communautaire en matière de maintien de l'ordre et de la sécurité en général et adopte à cette fin les mêmes options, à savoir la coopération intergouvernementale et la centralisation. Impliquant un net renforcement du pouvoir discrétionnaire de la police, la tendance sécuritaire, bien révélée par le Rapport officiel précité où il est précisé qu'un refus d'entrée peut se fonder sur " de simples renseignements selon lesquels [l'étranger] aurait causé des problèmes à la sûreté nationale ou aurait agi contre l'intérêt public " ${ }^{42}$, se manifeste par ailleurs en matière de santé publique. Car, bien que les dispositions de la loi de 1991 ne soient pas applicables aux étrangers atteints du SIDA, les rédacteurs du Rapport précité souhaitent l'élaboration d'études au niveau européen pour que les étrangers soient obligés de présenter un certificat médical attestant qu'ils ne sont pas séropositifs ${ }^{43}$. La tendance libérale Quoique d'étendue limitée, l'apparition de cette tendance marqua un tournant de la législation grecque sur l'immigration impliquant, d'une part, l'adoption d'une politique sociale pour les immigrés en situation régulière et, d'autre part, la reconnaissance de certains droits aux étrangers menacés d'expulsion administrative ainsi que l'établissement d'un ensemble de contraintes imposées aux autorités en matière d'expulsion. Il faut pourtant souligner que l'étendue limitée de cette tendance libérale est, en partie certes, contrebalancée par l'attitude tolérante adoptée souvent par la police vis-à-vis des étrangers clandestins. Cette attitude, incontestablement liée au coût financier des opérations policières en matière d'éloignement des étrangers du territoire, obéit aussi à des logiques de nature économique, comme nous l'avons déjà mentionné, ou politique, comme nous verrons plus loin. Ceci dit, nous estimons qu'elle ne saurait être en même temps dissociée de la longue expérience des Grecs en matière d'émigration ainsi que de persécutions subies, tout au long du vingtième siècle, tant à l'étranger que dans leur propre pays. Le souci de la protection de certains intérêts Nos observations ci-dessus, valables quant à l'interprétation de la politique adoptée à l'égard des 
immigrants étrangers, ne sauraient pourtant interpréter de manière satisfaisante la politique adoptée à l'égard des immigrants albanais. Celle-ci est en fait étroitement liée à l'évolution des relations gréco-albanaises en général et à la protection de la minorité grecque en Albanie en particulier. En effet, dès 1987, la Grèce a cherché à devenir le partenaire privilégié de l'Albanie. Elle a donc favorisé son ouverture sur le monde extérieur, appuyé sa participation aux différentes conférences régionales, renforcé les échanges commerciaux bilatéraux, envoyé des crédits importants et essayé de promouvoir, en tant que membre de l'Union européenne, le processus de réformes économiques albanaises. Ce renforcement des relations visant, bien évidemment, à un apaisement des tensions dans la région et notamment à l'amélioration du sort de la minorité grecque, objet de litige entre les deux pays, la Grèce a vu d'un très mauvais œil l'adhésion de l'Albanie, en 1992, à l'Organisation de la Conférence Islamique ${ }^{44}$ qui, allant de pair avec la formation de relations étroites, sur le plan économique, politique et militaire, entre Tirana et Ankara ${ }^{45}$ impliquait, d'une part, le renforcement de l'influence turque dans la région et, d'autre part, l'émergence éventuelle d'un axe musulman dans les Balkans. Sans vouloir alors minimiser les difficultés de contrôle dues à la morphologie des frontières, nous estimons que l'ampleur actuelle de l'immigration albanaise est en partie attribuable à la prise en considération des paramètres ci-dessus, les immigrants albanais étant en quelque sorte instrumentalisés par le gouvernement grec. Plus précisément, les rentrées en devises que procurent les émigrés albanais, estimées à 400-500 millions de dollars pour l'année $1993^{46}$, constituent le revenu principal de beaucoup de familles et correspondent à près d'un quart du PIB du pays ${ }^{47}$. Une grande partie de ces émigrés se trouvant en Grèce, les rentrées en devises procurées par ces derniers sont à présent estimées à 400 millions de dollars ${ }^{48}$. Ceci étant, l'adoption d'une attitude tolérante à leur égard, lors de leur entrée et/ou de leur séjour, renforcerait la position de la Grèce auprès de l'Albanie et lui permettrait de mieux assurer la protection des droits de la minorité grecque tandis que toute atteinte portée à cette minorité susciterait des reconduites à la frontière massives. Le seul enjeu ouvertement admis étant le sort de la minorité grecque, nous constatons, par exemple, que le bannissement du parti politique de la minorité Omonoia et la fermeture de certaines écoles grecques ont été probablement liés aux

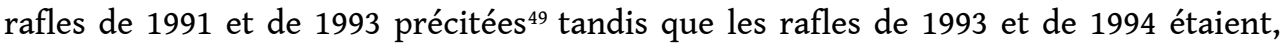
respectivement, des mesures de rétorsion contre l'expulsion d'un archimandrite grec et contre le déroulement d'un procès de cinq membres de la minorité grecque accusés de séparatisme ${ }^{50}$. En revanche, l'amélioration des relations gréco-albanaises, confirmée par la signature d'un traité d'amitié entre les deux pays en mars 1996, a été suivie par la déclaration du ministre des Affaires Étrangères que la Grèce envisageait d'accorder un statut particulier aux 200000 immigrants albanais présents sur son territoire. Conclusion Cette présentation des principaux aspects de la politique grecque en matière de contrôle de l'immigration a mis en évidence la complexité des logiques souvent contradictoires sous-tendant aussi bien sa conception que sa mise en œuvre. En effet, longtemps caractérisée par sa tendance répressive et son faible degré de cohésion et d'efficacité, cette politique a été modifiée en 1991 suivant trois logiques distinctes : le maintien de l'ordre et de la sécurité à l'intérieur du pays, mis en jeu par la forte hausse de l'immigration clandestine, l'harmonisation des options étatiques avec les lignes directrices dominantes en la matière au niveau communautaire et parmi les pays signataires des accords de Schengen et, en ce qui concerne le cas particulier des immigrants albanais, la protection de certains intérêts politiques et socio-économiques 
grecs. Les deux premières logiques étant caractérisées par de fortes affinités idéologiques, leur mise en œuvre a essentiellement impliqué un renforcement de la tendance sécuritaire. Mais la prise en considération de la troisième logique ainsi que, à un degré moindre, de certains facteurs d'ordre économique et humanitaire a fini par relativiser à plusieurs égards ce durcissement des réponses étatiques. Néanmoins, la création de " zones d'attente " pour les demandeurs d'asile et la future mise en place d'un corps de gardes frontaliers nous indique que, malgré l'apparition d'une tendance libérale, les options futures risquent d'être essentiellement influencées par la tendance sécuritaire. La prédominance de cette tendance au sein des options futures de la politique grecque devient enfin encore plus probable si nous prenons en considération, d'une part, le durcissement constant des mesures adoptées au niveau européen en la matière et, d'autre part, l'intensification des conflits locaux dans les Balkans et le Moyen Orient, qui est susceptible de provoquer la hausse de l'immigration clandestine et de la criminalité transfrontière ainsi que le renforcement des réseaux de trafic d'immigrés. Ceci étant, nous devons signaler que, assez paradoxalement, le processus d'européanisation et notamment la Convention d'application de l'accord de Schengen, que la Grèce doit ratifier avant ce mois d'octobre, n'ont pas un impact uniquement répressif sur la politique de contrôle de l'immigration grecque, puisqu'ils semblent produire également, quoiqu'indirectement, des effets libéraux. En effet, souhaitant adhérer à l'espace Schengen à partir d'une base assainie, le gouvernement grec a annoncé en janvier 1997 la régularisation imminente, sous certaines conditions, de tous les immigrés clandestins présents en Grèce. Il faut tout de même souligner que cette décision, qui n'est pas entièrement approuvée par les ministères de l'Ordre Public et de la Défense, n'a pas été encore appliquée, notamment à cause de la crise actuelle en Albanie.

\section{NOTES}

1. Anastassia Tsoukala est chercheur associé à l'Institut de Relations Internationales de l'Université Panteion d'Athènes.

2. Après l'Allemagne et le Luxembourg (Kathimerini, 2 décembre 1993, p. 8).

3. Kiriakatiki Eleftherotipia, 16 juin 1996, p. 64.

4. M. Baldwin-Edwards, " Immigration After 1992 ", Policy and Politics, 1991, 3, p. 203 ;

J. Condé, C. Taveres-Gravato, Les migrations internationales Sud-Nord (Grèce), OCDE, Paris, 1986, p. 8.

5. J.O. du 4 décembre 1991, A, 184.

6. Ministère de l'Ordre Public, Note d'information sur l'immigration clandestine (en grec), Athènes, 3 novembre 1996, p. 2.

7. X. Petrinioti, L'immigration en Grèce (en grec), Odysseas/Bibliothèque de l'Institut de Relations Internationales, Athènes, 1993, p. 41.

8. Cette diaspora est estimée à 400-650 000 personnes.

9. Eleftheros Tipos, 19 juin 1996, p. 27.

10. OCDE, Sopemi Report, 1993, p. 1 ; X. Petrinioti, op. cit., p. 19. 
11. H. Stark, " La question albanaise ", Politique étrangère, printemps 1994, p. 215 ; A. S. Wallden, "Les relations gréco-albanaises : politique et économie ", Courrier des pays de l'Est, 1993, 382, p. 60 ; X. Petrinioti, op. cit., p. 35.

12. OCDE, Sopemi Report, Paris, 1995, p. 26.

13. Eleftheros Tipos, 5 octobre 1995, p. 22.

14. Baldwin-Edwards M., op. cit., p. 201.

15. OCDE, 1993, op. cit., p. 12.

16. Ministère de l'Ordre Public, op. cit., p. 2.

17. R. Austin et al., " Albania's Greek Minority ", RFE/RL Research Report, 18 mars 1994, p. 20 ; A. S.Wallden, op. cit., p. 56 ; OCDE, 1993, op. cit., p. 12.

18. Ministère de l'Ordre Public, op. cit., p. 1 ; Eleftherotipia, 28 juillet 1994, p. 33.

19. Ministère de l'Ordre Public, op. cit., p. 1 ; Eleftherotipia, 28 juillet 1994, p. 33.

20. Rapport sur le contrôle des personnes aux passages routiers, ferroviaires ou autres ainsi qu'aux ports et aux aéroports utilisés comme points d'entrée et de sortie (en grec), ministère de l'Ordre Public, Athènes, mars 1992, pp. 46-47.

21. J.O. du 11 août 1994, A, 131. Sur le statut juridique antérieur des réfugiés, voir notamment Z. Papassiopi-Passia, " The legal condition of refugees in Greece ", Revue hellénique de droit international, 1994, pp. 341-376.

22. Ce qui plaçait la Grèce, pendant cette période, au troisième rang dans la Communauté, après le Danemark et les Pays-Bas, quant au taux de hausse annuelle des demandeurs d'asile (X. Petrinioti, op. cit., p. 29).

23. Le nombre d'étrangers auxquels a été reconnue la qualité de réfugié étant, dès 1987, en forte baisse, en 1996 cette politique gouvernementale a été critiquée par le HCR (Eleftherotipia, 15 février 1996, p. 15).

24. HCR, Note d'information (en grec), Athènes, juillet 1996, p. 2.

25. J.O. du 31 décembre 1996, A, 283.

26. Sur cette politique législative, voir A. Tsoukala, " La réforme du droit grec en matière d'immigration. L'entrée et l'éloignement des étrangers non communautaires ", Revue de science criminelle et de droit pénal comparé, 1994, 4, pp. 747-757.

27. Cette disposition est plus libérale que celle prévue par la législation antérieure, où l'entrée était en outre refusée à tout étranger atteint d'une maladie mentale.

28. Délivrée à cette fin par le service consulaire compétent.

29. J. Courtovic, "Le statut juridique des travailleurs migrants en Grèce " (en grec) in Fondation Marangopoulos pour les droits de l'homme, La protection des droits des travailleurs migrants et de leurs familles (en grec, en anglais et en français), Hestia, Athènes, 1994, p. 192.

30. En pratique, les tribunaux appliquent des peines de prison de quinze mois.

31. X. Petrinioti, op. cit., p. 26.

32. En juin 1993 ont été reconduits à la frontière près de 30000 immigrants albanais $(R$. Austin, " Albanian-Greek Relations : The Confrontation Continues ", RFE/RL Research Report, 20 août 1993, p. 33 ; H. Stark, op. cit., p. 215). En août 1994 ont été reconduits à la frontière environ 20000 Albanais, dont certains auraient été brutalisés par la police grecque (Le Monde, 28/29 août 1994, p. 4) et, à la fin du mois de septembre, ils ont atteint le chiffre de 51200 personnes - auxquelles se sont ajoutés 27500 immigrants qui se sont présentés de leur plein gré aux autorités.

33. Rapport sur le contrôle..., op. cit., p. 53, p. 66.

34. Idem, pp. 53-57.

35. Ibid, p. 53. 
36. V. Karydis, " The fear of crime in Athens and the construction of the "dangerous albanian " stereotype ", Chronika, 1995, 5, pp. 99-100.

37. Nous citons, à titre indicatif (en 1995) : meurtres : 27,2\%; vols à main armée : 50,6\% ; vols qualifiés : $27,9 \%$; faux et usage de faux : $32,5 \%$; viols : $20,4 \%$. Les Albanais incarcérés en Grèce dépassant, à la fin de 1995, les 700 personnes, en février 1996 Athènes a conclu avec Tirana un accord de transfert des prisonniers albanais en Albanie et a proposé, à cette fin, de couvrir une partie du coût nécessaire à l'accomplissement des travaux d'infrastructure du système pénitentiaire albanais (Kathimerini, 22 février 1996, p. 4).

38. Au début des années quatre-vingt-dix, la Grèce avait le taux de criminalité le plus bas dans l'Union européenne.

39. A. Tsoukala, " L'arrivée des immigrants en Grèce et leur intégration sociale ", intervention au séminaire de l'OCDE " Migrations, libre-échange et intégration régionale dans le Bassin méditerranéen ", Athènes, 30 octobre-1er novembre 1996 ; G. Voulgaris et al., " La perception de "l'autre" et les réactions suscitées par la présence de "l'autre" en Grèce aujourd'hui. Conclusions d'une recherche empirique " (en grec), Elliniki Epithéorissi Politikis Epistimis, 1995, 5, pp. 90-100 ; EKA, Tous différents, tous égaux. Enquête sur les immigrés clandestins et l'emploi (en grec), Athènes, 1995, pp. 51-95 ; Eurobarometer Trends, juin 1993, n³ 39, pp. A51-54 ; Tachidromos, 22 janvier 1992, pp. 40-50.

40. Sauf en cas d'hospitalisation urgente.

41. I. Psimmenos, L'émigration des Balkans. Exclusion sociale à Athènes (en grec), Glory Book/Papazissis, Athènes, 1995, pp. 160-173.

42. Rapport sur le contrôle..., op. cit., p. 13.

43. Idem, p. 21.

44. L. Zanga, " Albania Moves Closer to the Islamic World ", RFE/RL Research Report, 17 février 1993, pp. 28-31.

45. D. Hall, " Recent Developments in Greek-Albanian Relations ", Mediterranean Politics, 1996, pp. 99-100.

46. Economist Intelligence Unit, Country Report, First Quarter 1994 ; E. Lhomel, " Espoir d'un redressement économique ? ", La nouvelle alternative, 1994, 3, p. 18.

47. E. Lhomel, " L'économie albanaise en 1992 ", Courrier des pays de l'Est, 1993, 376, p. 58 ; E. Lhomel, " Espoir ... ", op. cit., p. 18 ; A. S. Wallden, op. cit., p. 50 ; D. Hall, op. cit., p. 90 .

48. O. Daniel, E. Lhomel, " L'Albanie en 1994-1995 : une stabilité précaire ", Notes et études documentaires, $\mathrm{n}^{\circ}$ 5027-28, 1996-2-3, p. 102.

49. L. Zanga, " Albanian-Greek Relations Reach a Low Point ", RFE/RL Research Report, 10 avril 1992, p. 20.

50. P. Papoudakis, " The Omonia-Five Trial : Democracy, Ethnic Minorities and the Future of Albania ", Südosteuropa, 1996, 4/5, pp. 342-358 ; R. Austin et al., op. cit., p. 21 ; H. Stark, op. cit., p. 215. 
INDEX

Mots-clés : contrôle, immigration

Index géographique : Grèce, méditerranée

Index chronologique : 1990 - 2000 